VOL. 9 (1973), 33-42.

\title{
Embedding near domains
}

\section{J.A. Graves and J.J. Malone}

\begin{abstract}
A class of near rings which generalizes the class of integral domains is defined. The definition of near domain is derived from the desirability of embedding near domains in near fields. The near domains presented here are shown to contain the $D$-rings of Berman and Siverman.
\end{abstract}

\section{Near domains}

We take integral domain to mean a commutative ring having no divisors of zero. Near rings with no zero divisors have been considered by Ligh and Malone [8], Clay [3] and Ferrero [4]. Ligh and Malone studied the properties of finite near rings having in one case no zero divisors and in another a finite number of zero divisors. Their work was not an attempt to generalize the concept of integral domain. Clay [3] called a near ring without divisors of zero a near integral domain but as he stated his definition was for the want of a better name. The main object of his note was the statement of the conjecture that, except for certain trivial cases, such a system having finite characteristic must have prime characteristic. This conjecture was later disproved by ferrero [4]. Thus the absence of zero divisors does not enhance the structure of a near ring to the extent one might expect.

THEOREM 1.1. Let $R$ be a near ring satisfying the right cancellation Zaw.

(i) For all $a \in R, \quad 0 a=a 0=0$.

(ii) $R$ has no (proper) zero divisors.

(iii) The left cancellation law holds in $R$. 
Proof. If $a \neq 0$ and $0 a=b \neq 0$, then $b=b b$ or $0 b=0 b b$ so that $0=0 b$ and $0=0 a$. If $a b=0, b \neq 0$, then $a b=0 b$ and $a=0$. If $a b=a c, a \neq 0$, then $a(b-c)=0$ and $b=c$. $/ /$

Ore has shown in [12] that, given a non-commutative ring having no proper divisors of zero, a necessary and sufficient condition for the ring to have a constructible division ring of quotients is that each pair of non-zero elements has a left (or right) common multiple.

DEFINITION 1.2. A near ring $R$ is said to satisfy the left (right) Ore condition if for all $\dot{a} \neq 0, b \neq 0 \in R$, there exist $s \neq 0$, $t \neq 0 \in R$ such that $s a=t b \cdot(a s=b t)$.

DEFINITION 1.3. A (left) near domain is a (left) near ring $D$ satisfying the left ore condition and the right cancellation law.

The usual proof that a finite integral domain is a field depends, essentially, only on right cancellation and may be used to establish:

THEOREM 1.4. If $R$ is a finite near ring for which the right cancelzation low holds, then $R$ is a near field.

It can be shown that finite cannot be omitted from the hypothesis of Theorem 1.4. Let $G$ be an additively written free group on two generators $x$ and $y$ and for each non-negative integer $n$ define $T_{n}: G \rightarrow G$ by $h(x, y) T_{n}=h(n x, n y)$, for all $h(x, y) \in G$. Let $R$ be the near ring generated additively by the $T_{n}$. McQuarrie [9] shows that $R$ is a distributively generated near ring with identity and Graves [5] shows that $R$ has the right cancellation property but satisfies neither the right nor the left Ore condition.

By Theorem 1.4, right cancellation implies the left Ore condition (when $R$ is finite). The near ring given as $2.5,26$ ) in [2] shows that the converse does not hold.

THEOREM 1.5. A near domain $R$ satisfying the descending chain condition on principal R-subgroups is a near field.

Proof. Ligh [7] has shown that a near ring satisfying the descending chain condition on principal $R$-subgroups and having no zero divisors must contain a left identity and moreover, if the left identity is unique, then $R$ is a near field. We have shown right cancellation implies no zero 
divisors. Hence an application of Ligh's result yields $e \in R$ such that $e r=r$, for all $r \in R$. If $a \neq 0 \in R$, we have for each $x \in R$, $x e a=x a$ which implies $x e=x$. Hence $e$ is the unique identity and, by Ligh's result, $R$ is a near field. //

\section{Near rings of quotients}

In this section we show that a near domain may be embedded in a near field and deduce some of the consequences of this embedding. Maxson [11] has defined near rings of quotients and stated conditions for a near ring to have a near ring of quotients. We generalize to the case of near rings of quotients with respect to a multiplicative set.

DEFINITION 2.1. Let $R$ be a near ring and $S$ a multiplicative set in $R$. We say $R$ satisfies the left ore condition with respect to $S$ if for each $(s, r) \in S \times R$ there exists $\left(s_{1}, r_{1}\right) \in S \times R$ such that $s_{1} r=r_{1} s$.

DEFINITION 2.2. Let $S$ be a multiplicative set in a near ring $R$. A near ring $R_{S}$ is called a near ring of left quotients of $R$ with respect to $S$ if

(i) $R_{S}$ has an identity 1 ,

(ii) there exists an embedding (monomorphism) $\Phi: R \rightarrow R_{S}$,

(iii) for each $s \in S, \Phi(s)$ is a unit in $R_{S}$,

(iv) every $q \in R_{S}$ can be expressed as $q=[\Phi(s)]^{-1}[\Phi(r)]$, $(s, r) \in S \times R$.

THEOREM 2.3. Let $S \neq \emptyset$ be a multiplicative set of (both left and right) cancellable elements in a (left) near ring $R$. Then a near ring of left quotients of $R$ with respect to $S$ exists if and only if $R$ satisfies the left Ore condition with respect to $S$.

Proof. Suppose $R_{S}$ exists. Then by the definition of $R_{S}$ there exists $\Phi: R \rightarrow R_{S}$ embedding $R$ into $R_{S}$. For each $(s, r) \in S \times R$, the product $[\Phi(r)][\Phi(s)]^{-1}$ in $R_{S}$ is expressible as 
$[\Phi(r)][\Phi(s)]^{-1}=\left[\Phi\left(s_{1}\right)\right]^{-1}\left[\Phi\left(r_{1}\right)\right]$, for some $\left(s_{1}, r_{1}\right) \in S \times R$. But then $\left[\Phi\left(s_{1}\right)\right][\Phi(r)]=\left[\Phi\left(r_{1}\right)\right][\Phi(s)]$ implies $s_{1} r=r_{1} s$ since $\Phi$ is a monomorphism.

To show the converse we construct $R_{S}$ by defining an equivalence relation " $~ "$ " on $S \times R$ and binary operations addition and multiplication an the set of equivalence classes $(S \times R) / \sim$. In proving this part of the theorem, there are many details to be verified and not all of these verifications will be given. However, several illustrations of the general techniques of the proof will be presented.

If $(c, x),(d, y) \in S \times R$ we define $(c, x) \sim(d, y)$ if there exists $(s, r) \in S \times R$ such that $s c=r d$ implies $s x=r y$. Note that even though $r$ may not be in $S, s c=r d$ where $s, c$ and $d$ are elements of $S$ implies the product $r d \in S$ and forces $r$ to be cancellable. From the definition, it can be noted that if $(c, x) \sim(d, y)$, then for every $\left(a_{1}, a_{2}\right) \in R \times R, a_{1} c=a_{2} d$ implies $a_{1} x=a_{2^{y}}$

Since the relation $\sim$ is an equivalence relation, we can consider the set of equivalence classes $(S \times R) / \sim=R_{S}$, where we denote the class coutanning $(c, x)$ by $x / c$, and equality of classes is determined by $x / c=y / d$ if and only if $(c, x) \sim(d, y)$.

Define + (addition) on $R_{S}$ by $x / c+y / d=(s x+r y) / s c=(s x+r y) / r d$ where $(6, r) \in S \times R$ satisfies $s c=r d \in S$.

We observe the definition is independent of the choice of $(s, r)$ by assuming $\left(s_{1}, r_{1}\right)$ satisfies $s_{1} c=r_{1} d$ and showing that $\left(s_{1} x+r_{1} y\right) / s_{1} c=(s x+r y) / s c$, that is, $\left(s_{1} c, s_{1} x+r_{1} y\right) \sim(s c, s x+r y)$. Let $\left(s_{2}, r_{2}\right)$ be such that $s_{2} s_{1} c=r_{2} s c$. Then $s_{2} s_{1}=r_{2} s$ and since $s_{1} c=r_{1} d, s c=r d$, we have, $s_{2} r_{1} d=s_{2} s_{1} c=r_{2} s c=r_{2} r d$ which implies $s_{2} r_{1}=r_{2} r$. Thus, $s_{2}\left(s_{1} x+r_{1} y\right)=s_{2} s_{1} x+s_{2} r_{1} y=r_{2} s x+r_{2} r y=r_{2}(s x+r y)$ implying the equivalence required.

Now addition is also well defined in the sense of being independent of 
the choice of the representatives for the equivalence classes. For let $(c, x) \sim\left(c^{\prime}, x^{\prime}\right)$ and $(d, y) \sim\left(d^{\prime}, y^{\prime}\right)$. We show $x / c+y / d=x^{\prime} / c^{\prime}+y^{\prime} / d^{\prime}$ by choosing $(s, r)$ and $\left(s^{\prime}, r^{\prime}\right)$ such that $s c=r d$ and $s^{\prime} c^{\prime}=r^{\prime} d^{\prime}$ to write $x / c+y / d=(s x+r y) / s c=(s x+r y) / r d$ and $x^{\prime} / c^{\prime}+y^{\prime} / d^{\prime}=\left(s^{\prime} x^{\prime}+r^{\prime} y^{\prime}\right) / s^{\prime} c^{\prime}=\left(s^{\prime} x^{\prime}+r^{\prime} y^{\prime}\right) / r^{\prime} d^{\prime}$. Then find $\left(s_{1}, r_{1}\right)$ such that $s_{1} s c=r_{1} s^{\prime} c^{\prime}=s_{1} r d=r_{1} r^{\prime} d^{\prime}$ whence $s_{1} s x=r_{1} s^{\prime} x^{\prime}$ and $s_{1} r y=r_{1} r^{\prime} y^{\prime}$, so that we have $s_{1}(s x+r y)=r_{1}\left(s^{\prime} x^{\prime}+r^{\prime} y^{\prime}\right)$ which yields $(s c, s x+r y) \sim\left(s^{\prime} c^{\prime}, s^{\prime} x^{\prime}+r^{\prime} y^{\prime}\right)$.

For any $e \in S, 0 / e$ is an additive right identity for $R_{S}$ and $-x / c$ is a right additive inverse for $x / c \in R_{S}$.

Since addition can be shown to be associative, we now have $\left(R_{S},+\right)$ is a group and, since addition on $R_{S}$ is defined in terms of the addition of $R,\left(R_{S},+\right)$ is abelian if and only if $(R,+)$ is abelian.

Let $x / c, y / d \in R_{S}$. Define - (multiplication) on $R_{S}$ by $x / c \cdot y / d=r y / s c$ where $(s, r) \in S \times R$ satisfies $s x=r d$.

This multiplication is independent of the choice of $(s, r)$ and is also independent of the choice of representatives of the equivalence classes.

For any $e \in S, e / e=I \in R_{S}$.

Now multiplication is associative; for let $x / c, y / d, z / e \in R_{S}$ and compute $(x / c \cdot y / d) \cdot z / e=r y / s c \cdot z / e=r_{1} z / s{ }_{1} s c$ where $s x=r d$ and $s_{1} r y=r_{1} e \cdot$ Also, $x / c \cdot(y / d \cdot z / e)=x / c \cdot r_{2} z / s_{2} d=r_{3} r_{2} z / s_{2} c$ where $s_{2} y=r_{2} e$, and $s_{3} x=r_{3} s^{d}$. To see that these products are equal we find $\left(s_{4}, r_{4}\right)$ such that $s_{4} s_{1} s c=r_{4} s_{3} c$, whence $s_{4} s_{1} s=r_{4} r_{3}$ and, using the equalities above, $s_{4} s_{1} r d=s_{4} s_{1} s x=r_{4} s_{3} x=r_{4} r_{3} s_{2} d$ which implies $s_{4} s_{1} r=r_{4} s_{3} s_{2}$. Further, $s_{4} r_{2} e=s_{4} s_{1} r y=r_{4} r_{3} s_{2} y=r_{4} r_{3} r_{2} e$ which implies $s_{4} r_{1}=r_{4} r_{3} r_{2}$. So $s_{4} r_{1} z=r_{4} r_{3} r_{2} z$ which says the desired equality holds. 
The fact that multiplication is left distributive over addition in $R_{S}$ follows from the left distributive property in $R$.

At this point we have shown $\left(R_{S},+, \cdot\right)$ is a left near ring with identity. To show $R_{S}$ is a near ring of left quotients of $R$ with respect to $S$ we must verify the remaining three defining properties of a near ring of left quotients.

Let $\Phi: R \rightarrow R_{S}$ be defined by $r \rightarrow$ er/e where $e \in S$. Then $\Phi$ is well defined because $e r / e=d r / d$ for all $e, d \in S$. For $r_{1}, r_{2} \in R$, $\Phi\left(r_{1}\right)+\Phi\left(r_{2}\right)=e r_{1} / e+e r_{2} / e=\left(e r_{1}+e r_{2}\right) / e=e\left(r_{1}+r_{2}\right) / e=\Phi\left(r_{1}+r_{2}\right)$ and $\Phi\left(r_{1}\right) \cdot \Phi\left(r_{2}\right)=e r_{1} / e \cdot e r_{2} / e=r e r_{2} / s e=\operatorname{ser}_{1} r_{2} / s e=e r_{1} r_{2} / e=\Phi\left(r_{1} r_{2}\right)$ where $(s, r)$ satisfies $\operatorname{ser}_{1}=r e$. So $\Phi$ is a near ring morphism. Further, kernel $\Phi=0$, for if $r \in R, \Phi(r)=e r / e=0 / e=e 0 / e$ implies $e r=e 0$ and so $r=0$. Thus $\Phi$ is a monomorphism embedding $R$ into $R_{S}$

For all $s \in S, \Phi(s)=$ es/e has an inverse $e / e s \in R_{S}$, for we compute, where $\left(s_{1}, r_{1}\right)$ satisfies $s_{1} e s=r_{1} e s$, that $e s / e \cdot e / e s=r_{1} e / s_{1} e=e / e=1$ since $e$ and $s$ cancellable implies $s_{1}=r_{1}$. Similarly, e/es $\cdot e s / e=e / e=1 \in R_{S}$.

Every $q=r / s \in R_{S}$ may be expressed as $[\Phi(s)]^{-1}[\Phi(r)]$ where $(s, r) \in S \times R$. Consider $[\Phi(s)]^{-1}[\Phi(r)]=e / e s \cdot e r / e=r_{1} e r / s_{1} e s=r / s$ where $\left(s_{1}, r_{1}\right)$ satisfies $s_{1} e=r_{1} e$, an element of $S$.

Thus $R_{S}$ is a near ring of left quotients of $R$ with respect to $S$ and the theorem is proved. //

Several points should be noted about the proof of Theorem 2.3. First, commutativity of addition in $R$ is not assumed. Secondly, the defining properties of a near ring of left quotients and the left Ore condition imply that any two near rings of left quotients for a given near ring $R$ are isomorphic. Thus we speak of the near ring of left quotients of $R$ 
with respect to $S$. Thirdly, as pointed out by Maxson [11], a parallel construction for near rings of right quotients does not hold because the right distributive law is necessary for addition in $R_{S}$ to be well defined, in the sense of being independent of the choice of $(s, r) \in S \times R$ used in the definition of addition.

DEFINITION 2.4. If $R$ is a near ring having at least one cancellable element and $C$ is the multiplicative set of all cancellable elements of $R$, then the near ring of left quotients of $R$ with respect to $C$ is called the total near ring of left quotients of $R$.

COROLLARY 2.5. The total near ring of left quotients of a near domain $D$ is a near field (called the near field of quotients of $D$ ).

Proof. We have shown that in $D$ right cancellation implies there are no zero divisors and this implies left cancellation. Hence the multiplicative set $S$ of all cancellable elements of $D$ is $D \backslash\{0\}$, so any nonzero element of the total near ring of left quotients has a multiplicative inverse. $/ /$

Since a near domain embeds in a near field, we have:

COROLLARY 2.6. Let $R$ be a near domain, then

(i) $(R,+)$ is commutative,

(ii) for all $a, b \in R, a(-b)=-(a b)=(-a) b$,

(iii) if $R$ has characteristic $m<\infty, m$ is prime.

We can observe by examples that neither of the defining properties of a near domain is by itself sufficient hypothesis to assure an embedding in a near field. The near ring given as $2.5,26$ ) in [2] is a near ring which satisfies the left Ore condition but which cannot be a sub near ring of a near field because it has zero divisors. Malcev [10] has given an example of a ring which has no divisors of zero but which is not embeddable in a division ring. Since Malcev's is a ring example, the absence of zero divisors along with right distributivity show that the right cancellation law holds. Thus his example provides a near ring satisfying the right cancellation law that is not embeddable in a near field.

The $D$-ring of Berman and Silverman [1] is the only embeddable (in a near field) generalization of integral domain appearing in the near ring 
literature. We now show near domains are generalizations of D-rings.

DEFINITION 2.7. A D-ring is a near ring $D$ such that

(i) $a b=0 ; a, b \in D$ implies $a=0$ or $b=0$,

(ii) for every $a \in D$ there are non-zero elements $a_{e}, a_{r} \in D$ such that $a_{e} a$ and $a a_{r}$ are in the multiplicative center of $D$.

THEOREM 2.8. If the near ring $R$ is a D-ring, then $R$ is a near domain.

Proof. First, $R$ satisfies left Ore condition (also right by similar proof). Let $a, b \in R$. Then there exists $a_{e} \neq 0 \in R$ such that $a_{e} a$ is in the multiplicative center of $R$, whence $\left(a_{e} a\right) b=b\left(a_{e} a\right)=\left(b a_{e}\right) a$. Thus there exist $x=a_{e} a$ and $y=b a_{e}$ in $R$ such that $x b=y a$, where $x$ and $y$ are non-zero if $a$ and $b$ are non-zero. Also, the right cancellation law holds in $R$. Let $a, b, c \neq 0 \in R$ satisfy $a c=b c$. There exists $c_{r} \neq 0 \in R$ such that $c c_{r}$ is in the multiplicative center and $c c_{p} \neq 0$ so that $a c c_{r}=b c c_{p}$, which implies $\left(c c_{p}\right) a=\left(c c_{p}\right) b$, so that $\left(c c_{n}\right)(a-b)=0$. Hence $a=b$ and $R$ is a near domain. //

For the ring of integers $Z$ (actually any Ore domain, see [6]), the field of quotients of any non-zero ideal of $Z$ is the same as that of $Z$, namely the rational numbers. Similarly we have:

THEOREM 2.9. Let $R$ be a near domain having $Q$ as its near field of quotients. If. $A \neq(0)$ is an ideal of $R$, then $Q$ is also the near fiezd of quotients of $A$.

Proof. We first observe that $A$ is in fact a near domain, so $A$ does have a near field of quotients and the statement of the theorem makes sense. Clearly $A$ satisfies the right cancellation law. Given $a \neq 0$, $b \neq 0$ in $A$, there exist $s \neq 0, t \neq 0$ in $R$ such that $s a=t b$. To find similar elements in $A$, take $x \neq 0 \in A$, whence $x s \neq 0$, xt $\neq 0$ are such that $(x s) a=(x t) b$ with $x s$ and $x t$ in $A$ since $A$ is an ideal. (Note that this actually shows any $R$-subgroup of a near domain is also a near domain.) 
Let $d / c \in Q$ and let $a \neq 0 \in A$. Then there exist $s \neq 0$, $t \neq 0 \in R$ such that $s c=t a \neq 0$, and further there exist $x \neq 0, y \in R$ such that $x s d=y a$. So $d / c=s d / s c=s d / t a=x s d / x t a=y a / x t a$, where $x t a \neq 0 \in A$ and $y a \in A$, by $A$ an ideal. Thus $Q$ is contained in the near field of quotients of $A$ and, since $A \subseteq R$, the theorem is proved. //

\section{References}

[1] Gerald Berman and Robert J. Silverman, "Near-rings", Amer. Math. Monthly 66 (1959), 23-34.

[2] James R. Clay, "The near-rings on groups of low order", Math. 2. 104 (1968), 364-371.

[3] James R. Clay, "A note on integral domains that are not right distributive", Elem. Math. 24 (1969), 40-41.

[4] G. Ferrero, "Stems planari e BIB-disegni", Riv. Mat. Univ. Parma 11 (1970), 1-16.

[5] James A. Graves, "Near domains", (Doctoral dissertation, Texas A\&M University, College Station, Texas, 1971).

[6] I.N. Herstein, Topics in ring theory (Chicago Lectures in Mathematics; The University of Chicago Press, Chicago, London, 1969).

[7] Steve Chong Hong Ligh, "On certain classes of near-rings", (Doctora] dissertation, Texas A\&M University, College Station, Texas, 1969).

[8] S. Ligh and J.J. Malone, Jr, "Zero divisors and finite near-rings", J. Austral. Math. Soc. 11 (1970), 374-378.

[9] B.C. McQuarrie, "A non-abelian near ring in which $(-1) r=r$ implies $r=0 "$, Canad. Math. Bulz. (to appear).

[10] A. Malcev, "On the immersion of an algebraic ring into a field", Math. Ann. 113 (1937), 686-691. 
[11] Carlton J. Maxson, "On near-rings and near-ring modules", (Doctoral dissertation, State University of New York at Buffalo, New York, 1967 ).

[12] Oystein Ore, "Linear equations in non-commutative fields", Ann. of Math. 32 (1931), 463-477.

El Paso Community College,

EI Paso,

Texas,

USA ;

Worcester Polytechnic Institute,

Worcester,

Massachussets,

USA. 